\title{
O PAPEL DA GESTÃO EM SAÚdE NA GERÊNCIA DE RESÍDUOS DE SERVIÇOS DE SAÚDE
}

\author{
THE ROLE OF hEALTH MANAGEMENT IN HEALTH SERVICES \\ WASTE MANAGEMENT
}

Kevin Fontelles Morais ${ }^{1}$

Liliane de Almeida Cardoso

\begin{abstract}
RESUMO: INTRODUÇÃO: resíduos de serviços de saúde podem ser caracterizados como aqueles que provém de estabelecimentos que oferecem serviços de assistência à saúde. O aumento da produção desses resíduos, pode ser explicado pelo crescimento do setor de saúde nas últimas décadas, levando em conta que o gerenciamento errôneo pode acarretar um ambiente favorável para a proliferação de múltiplos vetores e maior transmissão de doenças, além de contribuir para contaminação do meio ambiente, acidentes de trabalho e aumento de infecções hospitalares. OBJETIVO: analisar a importância da gestão em saúde no gerenciamento de resíduos de serviços de saúde. METODOS: foi realizada uma revisão integrativa de literatura, nas bases de dados: Scielo, BVS, LILACS e PubMed. Com um total de 11 artigos analisados entre os anos de 2000 a 2019, nos idiomas português, espanhol e inglês. RESULTADOS: foi evidenciado que há um desconhecimento dos profissionais de saúde e até mesmo dos gestores acerca do correto manejo dos resíduos de serviços de saúde acarretando gastos desnecessários e agravos ao meio ambiente. Além disso, também foi possível observar fragilidades dentro da própria gestão, como uma política ineficiente de gestão de resíduos de serviços de saúde, ausência de capacitação profissional e despreparo dos profissionais gestores. CONCLUSÃO: É necessário planejamentos e ações mais efetivas dentro do arcabouço das políticas voltadas para a gestão em saúde assim como também se enfatiza a importância da capacitação através do instrumento da educação permanente em saúde, para os profissionais e gestores de serviços de saúde.
\end{abstract}

Palavras chave: Resíduos de Serviço de Saúde. Gerenciamento de Resíduos. Gestão em Saúde.

\footnotetext{
1 Discente do Curso de Enfermagem da Universidade Federal de Campina Grande, kevinfontellesuf@gmail.com.

2 Discente do Curso de Enfermagem da Universidade Estadual da Paraíba, almeida.lilianne@gmail.com.
} 
RESUME: INTRODUCTION: healthcare waste can be characterized as that that comes from facilities that offer health care services. The increase in the production of this waste can be explained by the growth of the health sector in the last decades, considering that mismanagement can lead to a favorable environment for the proliferation of multiple vectors and greater disease transmission, besides contributing to the contamination of the health sector. environment, work accidents and increased hospital infections. OBJECTIVE: to analyze the importance of health management in health services waste management. METHODOLOGY: an integrative literature review was performed in the following databases: Scielo, VHL, LILACS and PubMed. With a total of 11 articles analyzed from 2000 to 2019, in Portuguese, Spanish and English. RESULTS: it was evidenced that there is a lack of knowledge of health professionals and even managers about the correct management of health services waste causing unnecessary expenses and harms to the environment. In addition, it was also possible to observe weaknesses within the management itself, such as an inefficientpolicy of waste management of health services, lack of professional training and unpreparedness of profession nal managers. CONCLUSION: more effective planning and actions are needed within the framework of policies for health management, as well as the importance of training through the instrument of permanent health education for health service professionals and managers.

Descriptors: Health Care Waste. Waste Management. Health Management. 


\section{INTRODUÇÃO}

Resíduos de serviços de saúde podem ser caracterizados como aqueles que provém de estabelecimentos que oferecem serviços de assistência à saúde - seja ela de caráter humano ou animal. Atualmente, é notável o aumento da produção de resíduos de serviços de saúde, fato que pode ser explicado pelo crescimento do setor de saúde nas últimas décadas (DIAS, 2017; OMS, 2014).

Atualmente, os resíduos de serviços de saúde são classificados em grupos de acordo com sua característica, sendo elas: Grupo A (resíduos infectantes, subdividese em A1, A2, A3, A4 e A5, conforme as características de cada resíduo), Grupo B (resíduos químicos), Grupo C (resíduos radioativos), Grupo D (resíduos comuns, incluindo neste grupo estão os resíduos considerados recicláveis), e Grupo E (resíduos perfuro cortantes) (BRASIL, 2004, CONAMA, 2005).

O gerenciamento dos resíduos de serviços de saúde deve apresentar programas que visem abranger o tratamento e a destinação final - seguindo as etapas de segregação, acondicionamento, coleta interna, armazenamento, coleta externa, tratamento de disposição final, sempre levando em conta as características de cada resíduo, sendo esse processo fundamental para a promoção da saúde e preservação do meio ambiente (DIAS, 2017; OLIVEIRA, 2014; BRASIL, 2004).

Levando em conta que o gerenciamento errôneo de resíduos de serviços de saúde pode acarretar um ambiente favorável para a proliferação de múltiplos vetores, é de se esperar que haja, portanto, uma maior transmissão de doenças, além de contribuir na contaminação do meio ambiente, acidentes de trabalho e no aumento de infecções hospitalares (TEIXEIRA, 2018; NUNES, 2012; DUTRA, 2011).

Atualmente, se faz necessário que todo gerador de resíduos de serviços de saúde elabore e implante um Plano de Gerenciamento dos Resíduos de Serviços de Saúde (PGRSS) em seu serviço, seguindo as orientações das Resoluções da Diretoria Colegiada da Agência Nacional de Vigilância Sanitária (ANVISA) n 306/04 e do Conselho Nacional do Meio Ambiente (CONAMA) n 358/05. 
O Plano de Gerenciamento dos Resíduos de Serviços de Saúde (PGRSS) consiste num documento que visa o correto manejo dos resíduos gerados pelos serviços de saúde, com o objetivo de minimizar a produção desses resíduos e proporcionar um encaminhamento seguro, levando em conta à proteção dos trabalhadores e à preservação do meio ambiente (CARVALHO, 2016; GOMES, 2014).

Porém como Moreira (2012) analisa, tanto no Brasil como em outros países em desenvolvimento, as exigências legais com respeito ao gerenciamento dos resíduos ainda não estão sendo cumpridas criteriosamente pelos serviços de saúde e que o Plano de Gerenciamento dos Resíduos de Serviços de Saúde, mesmo sendo claro e objetivo com relação ao cumprimento das exigências estabelecidas, não é suficiente para aprimorar a gestão.

Este problema pode ser relacionado à falta de estabelecimento de planejamento estratégico e operacional, à ausência de definição de planos de ação curto, médio e longo prazo, e, sobretudo ao não envolvimento do corpo funcional da instituição, além da ausência de estabelecimento de indicadores e metas de acompanhamento (SANTOS, 2015).

Partindo do exposto, o trabalho tem por objetivo analisar a seguinte questão: "Qual a importância da gestão em saúde no gerenciamento de resíduos de serviços de saúde na literatura científica?

\section{METODOLOGIA}

Trata-se de uma revisão integrativa de literatura (RIL) de caráter qualitativo, construída a partir de busca controlada de dados secundários em artigos científicos indexados nas bases de dados: Scientific Eletronic Library Online (SciELO), Literatura Latino-americana e do Caribe em Ciências da Saúde (LILACS); National Library of Medicine (PubMed) e na Biblioteca Virtual de Saúde (BVS), isto a partir do uso dos descritores em saúde: "Resíduos de Serviço de Saúde", "Gerenciamento de Resíduos"; "Gestão em Saúde". 
A busca dos dados foi conduzida com foco na seguinte questão norteadora "qual a importância da gestão em saúde no gerenciamento de resíduos de serviços de saúde na literatura científica?". Para triagem dos artigos utilizou-se os seguintes critérios de inclusão: resultados publicados entre 2003 e 2019; idiomas português, espanhol e inglês; bem como materiais complementares disponíveis online, totalizando 11 documentos científicos analisados no estudo.

A coleta das informações ocorreu entre os meses de maio e junho de 2019, mediante uso de formulário estruturado, adaptado (URSI, 2005) para as variáveis necessária à questão norteadora, apresentando-se conforme Quadro 1: identificação das publicações, características metodológicas dos estudos e conclusões.

A apresentação dos resultados e discussão dos dados obtidos foi feita de forma descritiva possibilitando ao leitor a avaliação da aplicabilidade da revisão integrativa elaborada, de forma que fosse possível fornecer subsídios ao profissional da saúde em sua tomada de decisão cotidiana.

\section{DESENVOLVIMENTO}

A Gestão em Saúde pode ser caracterizada como "o conhecimento aplicado no manejo do complexo das organizações de saúde, envolvendo a gerência de redes, esferas públicas de saúde, hospitais, laboratórios, clínicas e demais instituições e serviços de saúde". Abrange ainda três grandes dimensões: os espaços dos cuidados diretos- singulares e multiprofissionais; as diversas instituições de saúde; e a exigência da formação e operação de redes de serviços de saúde para uma assistência universal, integral, equânime, de qualidade e eficiente para as necessidades de saúde da população (LORENZETTI, 2014).

É recorrente a constatação de que a gestão em saúde ainda está ancorada em métodos e estratégias tradicionais, oriundas da teoria clássica da administração. E que construir novas formas de gestão na área da saúde, fundadas na participação, práticas cooperativas e interdisciplinares onde trabalhadores e usuários atuem como sujeitos ativos, permanece como desafio (MATOS, 2006). 
Atualmente é observável o crescimento progressivo dos gastos em saúde em todo o mundo, corroborado para o fato de que os métodos tradicionais de gestão estão longe de gerar uma performance eficaz e eficiente nos resultados dos serviços de saúde. A Organização Mundial da Saúde estima que 20\% a 40\% do gasto total em saúde é desperdiçado por diversas formas de ineficiência (OMS, 2010).

No Brasil, a partir dos anos 1980, com a Constituição de 1988 e a criação do Sistema Único de Saúde (SUS), as ações e serviços de saúde foram institucionalizados como política pública, institucionalizando a saúde como um direito de todos e um dever do Estado. O destaque da questão da gestão, como um dos aspectos estruturais dos desafios para a transformação positiva da situação de saúde no Brasil é de suma importância, levando em consideração que a Gestão é um ponto chave na administração de qualquer serviço ou organização de saúde.

\section{RESULTADOS E DISCUSSÃO}

Os resultados da RIL foram organizados de acordo com os componentes estruturantes dos artigos, de forma a delinear com clareza as variáveis de interesse. 


\begin{tabular}{|c|c|c|c|}
\hline Titulo do Artigo & Autores & Objetivo Principal & Conclusão \\
\hline $\begin{array}{l}\text { Análise da taxa de geração de } \\
\text { residuos de serviços de saúde } \\
\text { em um hospital universitário; }\end{array}$ & $\begin{array}{l}\text { DIAS GL; SARTURI F; } \\
\text { CAMIPONOGARA S; }\end{array}$ & $\begin{array}{l}\text { Identificar as taxas de geração } \\
\text { de residuos por grupo, e avaliar } \\
\text { a influência da sazonalidade } \\
\text { sobre a geração de RSS de um } \\
\text { Hospital Universitário do interior } \\
\text { do Estado do Rio grande do Sul; }\end{array}$ & $\begin{array}{l}\text { Há uma maior atenção aos } \\
\text { residuos do grupo A e uma } \\
\text { negligência dos demais grupos } \\
\text { que poderiam ser reciclados. }\end{array}$ \\
\hline $\begin{array}{l}\text { Análise da gestão e } \\
\text { gerenciamento dos residuos } \\
\text { de serviços de saúde (RSS) } \\
\text { do Hospital de Emergência de } \\
\text { Macapá, Amapá, Brasil }\end{array}$ & $\begin{array}{l}\text { MADERS, G.R; } \\
\text { CUNHA. H.F.A. }\end{array}$ & $\begin{array}{l}\text { Produzir informações sobre os RSS } \\
\text { no hospital de pronto socorro do } \\
\text { estado: o Hospital de Emergência, } \\
\text { localizado na capital Macapá }\end{array}$ & $\begin{array}{l}\text { Há inexecução do Plano de } \\
\text { Gerenciamento dos Residuos dos } \\
\text { Serviços de Saúde (PGRSS) do } \\
\text { Hospital, traduzida na falta de } \\
\text { atitudes gerenciais positivas e } \\
\text { educação continuada, somada à } \\
\text { inoperância dos órgãos de } \\
\text { fiscalização são as causas desse } \\
\text { cenário. }\end{array}$ \\
\hline $\begin{array}{l}\text { Avaliação da Gestão dos } \\
\text { Residuos em Unidades Básicas } \\
\text { de Saúde de um Municipio Sul- } \\
\text { brasileiro }\end{array}$ & $\begin{array}{l}\text { TEIXEIRA MV, } \\
\text { ECHEVARIA-GUANILO } \\
\text { ME, KNUTH FG, et al }\end{array}$ & $\begin{array}{l}\text { Analisar a gestão dos residuos de } \\
\text { serviços de saúde e, o conhecimento } \\
\text { dos profissionais de enfermagem } \\
\text { acerca da legislação vigente. }\end{array}$ & $\begin{array}{l}\text { As unidades apresentam } \\
\text { importantes fragilidades no } \\
\text { processo de gerenciamento dos } \\
\text { Residuos Sólidos de Saúde. }\end{array}$ \\
\hline $\begin{array}{l}\text { Gestão em Saúde no Brasil: } \\
\text { Diálogo com Gestores } \\
\text { Públicos e Privados }\end{array}$ & $\begin{array}{l}\text { LORENZETTI } J \text {, } \\
\text { LANZONI GMM, et al }\end{array}$ & $\begin{array}{l}\text { Identificar os principais problemas } \\
\text { da gestão em saúde no Brasil, a } \\
\text { partir da opinião de gestores } \\
\text { escolhidos intencionalmente. }\end{array}$ & $\begin{array}{l}\text { O SUS carece de mais recursos, } \\
\text { financiamento estável e de } \\
\text { gestão capaz de transformar } \\
\text { seus principios e diretrizes em } \\
\text { realidade. }\end{array}$ \\
\hline $\begin{array}{l}\text { Plano De Gerenciamento De } \\
\text { Residuos De Saúde: } \\
\text { Identificando A Opinião Da } \\
\text { Equipe De Um Ambulatório. }\end{array}$ & $\begin{array}{l}\text { CARVALHO MF, } \\
\text { PEREIRA LR, } \\
\text { NASCIMENTO E. et al }\end{array}$ & $\begin{array}{l}\text { Analisar as opiniões da equipe de } \\
\text { um ambulatório quanto às etapas } \\
\text { de construção de um Plano de } \\
\text { Gerenciamento de Residuos de } \\
\text { Serviço de Saúde }\end{array}$ & $\begin{array}{l}\text { Implantar o PGRSS em unidades } \\
\text { de saúde ê de extrema } \\
\text { importância, principalmente por } \\
\text { possibilitar a redução de } \\
\text { impactos ambientais e o } \\
\text { cumprimento de normas de } \\
\text { segurança. }\end{array}$ \\
\hline $\begin{array}{l}\text { Residuos dos serviços de } \\
\text { saúde: desafios e perspectivas } \\
\text { na atenção primária. }\end{array}$ & $\begin{array}{l}\text { OLIVEIRA LL, } \\
\text { SOUZA PM, et al }\end{array}$ & $\begin{array}{l}\text { Objetivou-se analisar o gerenciamento } \\
\text { dos residuos sólidos produzidos em } \\
\text { unidades básicas de saúde da } \\
\text { familia, desde a geração até a } \\
\text { disponibilização para coleta externa. }\end{array}$ & $\begin{array}{l}\text { Evidencia-se a } \\
\text { necessidade de um plano de } \\
\text { gerenciamento dos residuos } \\
\text { capaz de ser ajustado à realidade } \\
\text { dos serviços de saúde } \\
\text { observando as normas. }\end{array}$ \\
\hline $\begin{array}{l}\text { Gerenciamento de residuos } \\
\text { sólidos em um hospital de } \\
\text { ensino em Brasilia }\end{array}$ & $\begin{array}{l}\text { DUTRA, LMA; } \\
\text { MONTEIRO, PS }\end{array}$ & $\begin{array}{l}\text { Analisar a dinâmica do } \\
\text { gerenciamento dos Residuos de } \\
\text { Serviço de Saúde RSS) sólidos, no } \\
\text { Hospital Regional da Asa Norte } \\
\text { (HRAN) e verifcar se as práticas de } \\
\text { manuseio destes residuos, por } \\
\text { membros das equipes de enfermagem } \\
\text { e de higienização, estão de acordo às } \\
\text { normas e legislação pertinentes. }\end{array}$ & $\begin{array}{l}\text { A quantidade de residuos } \\
\text { produzida está de acordo com a } \\
\text { realidade da América Latina. } \\
\text { As etapas do manejo dos } \\
\text { RSS, em todas as unidades não } \\
\text { estavam atendendo aos } \\
\text { processos opcracionais } \\
\text { recomendados. Verifcou-se que } \\
\text { o conhecimento sobre o manejo } \\
\text { do RSS, pelas diferentes } \\
\text { categorias profssionais, era } \\
\text { limitado ou não possuiam } \\
\text { informação sobre o assunto. }\end{array}$ \\
\hline $\begin{array}{l}\text { Gerenciamento de residuos } \\
\text { de serviços de saúde: uma } \\
\text { revisão de literatura }\end{array}$ & $\begin{array}{l}\text { NUNES TSP, } \\
\text { GUTEMBERG } \\
\text { ACB, et al }\end{array}$ & $\begin{array}{l}\text { Identificar a relação do gerenciamento } \\
\text { dos residuos de serviços de saúde com } \\
\text { a saúde do trabalhador, mediante } \\
\text { revisão de literatura e discutir as } \\
\text { implicações do gerenciamento de } \\
\text { residuos para a enfermagem. }\end{array}$ & $\begin{array}{l}\text { Os autores são unânimes em } \\
\text { ressaltar a necessidade de } \\
\text { capacitar os profissionais de } \\
\text { saúde para o correto } \\
\text { gerenciamento dos } \\
\text { residuos, pois grande parte } \\
\text { dos profissionais não sabe } \\
\text { o que são residuos e não } \\
\text { valoriza o correto manuseio } \\
\text { dos mesmos na prática } \\
\text { hospitalar. }\end{array}$ \\
\hline
\end{tabular}




\begin{tabular}{|c|c|c|c|}
\hline Título do Artigo & Autores & Objetivo Principal & Conclusão \\
\hline $\begin{array}{l}\text { Conhecimento de enfermeiros } \\
\text { da estratégia Saúde da familia } \\
\text { sobre residuos dos serviços de } \\
\text { saúde; }\end{array}$ & $\begin{array}{l}\text { SANTOS MA, } \\
\text { SOUZA AO; }\end{array}$ & $\begin{array}{l}\text { Avaliar o conhecimento de } \\
\text { enfermeiros da ESF sobre RSS }\end{array}$ & $\begin{array}{l}\text { Apesar da existência de um } \\
\text { significativo conhecimento } \\
\text { sobre o tema, ainda há } \\
\text { necessidade de trabalhos de } \\
\text { conscientização e } \\
\text { desenvolvimento de práticas } \\
\text { adequadas de gerenciamento } \\
\text { de Residuos de Serviços de } \\
\text { Saúde. }\end{array}$ \\
\hline $\begin{array}{l}\text { Estudo descritivo sobre o } \\
\text { gerenciamento de residuos de } \\
\text { serviços de saúde no } \\
\text { municipio de Jatai, }\end{array}$ & $\begin{array}{l}\text { MELO CP, } \\
\text { BARBOSA LB et al }\end{array}$ & $\begin{array}{l}\text { Descrever o gerenciamento de } \\
\text { residuos de serviços de saúde } \\
\text { (RSS) no municipio de Jatai, } \\
\text { Estado de Goiás, Brasil }\end{array}$ & $\begin{array}{l}\text { O gerenciamento de RSS na } \\
\text { área do estudo foi } \\
\text { considerado inadequado } \\
\text { segundo as normas } \\
\text { regulamentadoras vigentes. }\end{array}$ \\
\hline $\begin{array}{l}\text { Biossegurança e residuos } \\
\text { de serviços de saúde no } \\
\text { cotidiano acadêmico }\end{array}$ & $\begin{array}{l}\text { GOMES LC, } \\
\text { MIGUEL YD et al }\end{array}$ & $\begin{array}{l}\text { Avaliar o conhecimento da } \\
\text { comunidade acadêmica do } \\
\text { campus III - Jardim } \\
\text { Botânico, da Universidade } \\
\text { Federal do Paraná (UFPR) } \\
\text { sobre Biossegurança e } \\
\text { Residuos de Serviços de Saúde } \\
\text { (RSS). }\end{array}$ & $\begin{array}{l}\text { A pesquisa evidenciou uma } \\
\text { lacuna no conhecimento e na } \\
\text { aplicação de medidas } \\
\text { biosseguras e responsáveis. }\end{array}$ \\
\hline
\end{tabular}

Quadro 1: Descrição da amostra da RIL. Fonte: Dados da Pesquisa, 2019.

\section{Ausência de Capacitação}

Em sua pesquisa, Teixeira (2018) observa que $57,8 \%$ dos profissionais entrevistados relataram não conhecer a legislação acerca dos RSS, no entanto, todos se referiram produtores de resíduos em seus locais de trabalho. Isso se confirma ao analisarmos que todos os profissionais conhecem os tipos de resíduos gerados em seu ambiente de trabalho, porém, muitos desconhecem a legislação. Fatos como este podem aumentar os riscos de descarte e manuseio inadequado dos resíduos.

Além disso foi possível observar que todos os profissionais entrevistados afirmaram conhecer os resíduos gerados, a maioria deles $(89,5 \%)$ informaram realizar a segregação desses materiais, porém $73,7 \%$ afirmaram não conhecer a classificação dos RSS. Somando-se a isso, $57,8 \%$ não conheciam a legislação dos resíduos de serviços de saúde e $36,8 \%$ dos profissionais desconheciam os cuidados 
específicos para cada tipo de resíduo. Esses dados contraditórios sugerem um gerenciamento dos RSS ineficaz, uma vez que os profissionais poderiam estar descartando os resíduos de forma inadequada (TEIXEIRA, 2018).

Ainda, em uma pesquisa realizada em 15 instituições de saúde do município de Jataí-GO, demonstrou que não houve capacitação dos funcionários das instituições analisadas para o manejo dos resíduos gerados, capacitação que constitui uma exigência das normas regulamentadoras vigentes (MELO, 2013).

Em um estudo realizado na cidade de Campina Grande - PB, foi possível destacar que estabelecimentos de saúde integrantes da atenção básica geram resíduos dos diversos grupos classificáveis - A, B, D e E, que não estão sendo corretamente acondicionados e segregados. Este fato foi explicado pela ausência de dois fatores importantes: inexistência de treinamento e a ausência de acondicionadores em quantidade e/ou qualidade necessárias (OLIVEIRA, 2014).

Faz-se mister enfatizar que a segregação inadequada dos resíduos, muitas vezes, está vinculada à ausência de treinamento, o que acarreta em uma não efetivação do PGRSS já que, sem o treinamento, o profissional, muitas vezes, não terá o conhecimento real do risco trazido pelo contato ou descarte inadequado dos resíduos contaminados, fato que corrobora para a importância da presença de políticas de educação permanente em saúde incentivadas pela gestão do serviço (CARVALHO, 2016).

\section{Fragilidades}

Um estudo realizado no Hospital de Emergência de Macapá voltado para a análise da gestão e gerenciamento dos resíduos de serviços de saúde revelou que a legislação sobre o assunto é conhecida de maneira superficial por gestores e funcionários, assim como também há uma ausência na execução das atribuições necessárias no manejo integrado dos resíduos. Além disso, também foi evidenciado que o PGRSS não é atualizado e não há uma capacitação dos profissionais quanto ao assunto (MADERS, 2015). 
A adoção de práticas responsáveis, o investimento na melhoria do próprio sistema-infraestrutura, envolvimento da comunidade hospitalar por processos de capacitação, a implementação de medidas de reciclagem e o correto acondicionamento e segregação de resíduos de serviços de saúde são mecanismos que possibilitariam uma redução em cerca de $80 \%$ nos gastos com tratamento e disposição final dos mesmos, recursos estes que poderiam ser úteis para a melhoria do próprio serviço (MADERS, 2015).

Lorenzetti (2014) ao dialogar com gestores de saúde - âmbito público ou privado, destacou como principais fragilidades: o despreparo dos profissionais para o exercício da administração, lentidão na incorporação de novas tecnologias de informação e processos de gestão e de organização do trabalho. Além disso, foram identificadas barreiras de legislação que restringem a agilidade necessária, a alta rotatividade dos gestores das esferas federativas em função da relação com os processos partidários e eleitorais, gerando descontinuidade, permanentes recomeços e desmotivação dos profissionais e trabalhadores.

\section{Incremento de Ações/Estratégias}

Dias (2017) verificou que há uma centralização de estudos e ações voltadas para resíduos considerados infectantes - grupo $A$, negligenciando resíduos integrantes de grupos tido como recicláveis e comuns - D e D-recicláveis, que inclusive são utilizados por famílias que retiram sua fonte de renda a partir da coleta e venda desses resíduos, corroborando para o fato de que a segregação desses resíduos de serviços de saúde é de suma importância e apresenta um impacto importante não só no âmbito da saúde pública, mas também em questões sociais.

Além disso, o gestor deve ter o entendimento de que os estabelecimentos de saúde devem contar com recipientes apropriados para acondicionar cada tipo de resíduo, garantir identificação apropriada do material, facilitar as operações de transporte e limpeza. Ainda, devem ser herméticos para evitar exposições desnecessárias e estar integrados às condições físicas e arquitetônicas do local. 
Esses recipientes são complementados com o uso de sacos plásticos específico para efetuar uma embalagem adequada dos resíduos (OLIVEIRA, 2014).

Também se faz importante enfatizar que o acondicionamento é uma etapa indispensável em um plano de manejo de resíduos de serviços de saúde, uma vez que possibilita a prevenção de contato com os seres vivos e o meio ambiente. Em contrapartida, o mau acondicionamento desses resíduos provoca a contaminação de produtos que poderiam ser reciclados, aumentando, portanto, a quantidade de resíduos considerados infectados (TEIXEIRA, 2018).

A correta distinção dos resíduos, assim como o correto descarte proporcionam um acondicionamento coerente, com menores riscos à saúde dos trabalhadores e ao meio ambiente; somando-se a isso, a diminuição dos gastos financeiros com possíveis resíduos descartados de maneira errônea (SANTOS, 2012).

\section{CONCLUSÃO}

Portanto é conclusivo que a destinação final dos resíduos de serviços de saúde deve ser um ponto prioritário no processo de gestão de qualquer serviço voltado para a atenção à saúde, processo esse que deve ser planejado e implementado conforme as diretrizes nacionais estabelecidas pela ANVISA e pelo CONAMA.

Durante a pesquisa, foi possível evidenciar problemas relacionados à própria gestão do serviço que corroboram para gastos desnecessários oriundos do mal planejamento. Dentre os problemas destacam-se, a ausência de um plano formal para o gerenciamento dos resíduos de saúde (PGRSS), ou gerenciamento assistemático e a não capacitação dos profissionais de saúde acerca do manejo dos resíduos e o coordenador da unidade desconhece tanto a legislação quanto o PGRSS.

A determinação e quantificação dos resíduos oriundos de serviços de saúde se faz um importante mecanismo de contribuição na gestão em saúde, visto que seu adequado planejamento ameniza os impactos ambientais e danos à saúde pública, 
assim como também contribui na diminuição de gastos e possibilita uma melhoria na qualidade da assistência do serviço.

Além disso, foi possível observar que há um desconhecimento dos profissionais e dos próprios gestores acerca do assunto, fato que corrobora para a importância do incremento de ações de educação permanente em saúde nos serviços.

\section{REFERÊNCIAS BIBLIOGRÁFICAS}

BRASIL, Agência Nacional de Vigilância Sanitária. Resolução da Diretoria Colegiada $n^{\circ} \mathbf{3 0 6}$, de 7 de dezembro de 2004. Dispõe sobre o Regulamento Técnico para o gerenciamento de resíduos de serviços de saúde. Diário Oficial [da] República Federativa do Brasil. Brasília, DF, Seção 1, p. 49, 2004.

CARVALHO, M. F; PEREIRA, L. R; NASCIMENTO, E; ANDRADE, R. D. Plano De Gerenciamento De Resíduos De Saúde: Identificando A Opinião Da Equipe De Um Ambulatório. Revista de Enfermagem UFPE on line. Recife, v.10, n.5, p.4304-4310, 2016.

CONAMA. Conselho Nacional do Meio Ambiente. Resolução $n^{\circ}$ 358, de 29 de abril de 2005. Dispõe sobre o tratamento e disposição final dos resíduos de serviço de saúde e dá outras providências. Diário Oficial [da] República Federativa do Brasil, Brasília, DF, Seção 1, p. 63-65, 2005.

DIAS, G. L; SARTURI, F; CAMPONOGARA, S; LIMA, S. B. S; LOPES, L.F. D; TREVISAN, C.M. Análise da taxa de geração de resíduos de serviços de saúde em um hospital universitário. Revista Fundamental Care Online. v.9, n. 1, p.96-98, 2017.

DUTRA, L. M. A; MONTEIRO, P. S. Gerenciamento de resíduos sólidos em um hospital de ensino em Brasília. Comunicação em Ciências da Saúde. v. 22, n. 4 p.305-314, 2011.

GOMES, L. C; MIGUEL, Y. D; ROCHA, T. C; GOMES, E. C. Biossegurança e resíduos de serviços de saúde no cotidiano acadêmico. Revista de Ciências Farmacêuticas Básica e Aplicada. v.35, n.3, p. 443-450, 2014.

LORENZETTI, J; LANZONIG. M. M; ASSUITI, L. F. C; PIRES, D. E. P; RAMOS, F. R. S. Gestão em Saúde no Brasil: Diálogo com Gestores Públicos e Privados. Texto Contexto Enfermagem.v.23, n.2, p.417-425, 2014.

MADERS, G. R; CUNHA, H. F. A. Análise da gestão e gerenciamento dos resíduos de serviços de saúde (RSS) do Hospital de Emergência de Macapá, Amapá, Brasil. Revista de Engenharia Sanitária e Ambiental. v.20, n.3, p. 379-388, 2015.

MELO, C. P; BARBOSA, L. B; SOUZA, M. R; BARCELOS, I. S. C. Estudo descritivo sobre o gerenciamento de resíduos de serviços de saúde no município de Jataí, Goiás, 2010. Revista Epidemiologia e Serviço de Saúde. v.22, n.3, p.517-524, 2013.

MOREIRA, A. M. M. Gerenciamento de resíduos de serviços de saúde: um desafio para unidades básicas de saúde. Dissertação (Mestrado em Ciências). Faculdade de Saúde Pública, Universidade de São Paulo, São Paulo, SP. Brasil, 2012. 
NUNES, T. S. P; GUTEMBERG, A. C. B; ARMANDO, C. B; PINTO, F. F; LEMOS, M. C; PASSOS, J. P. Gerenciamento de resíduos de serviços de saúde: uma revisão de literatura. Revista de Pesquisa: Cuidado é Fundamental Online. (Ed. Supl.), p. 57-60, 2012.

OLIVEIRA, L..L; SOUZA, P. M; CLEMENTINO, F. S; PAIVA, S. C; ROCHA, F. D. L. J. Resíduos dos serviços de saúde: desafios e perspectivas na atenção primária. Revista de Enfermagem UERJ. v.22, n.1, p.29-34, 2014.

OMS. Organização Mundial da Saúde, 2010. Disponível em: http://www. who.int/iris/bitstream/10665/85349/1/9789241548564_eng.pdf?. Acesso em: 20/09/19.

SANTOS, M. A; SOUZA, A. O. Conhecimento de enfermeiros da Estratégia Saúde da Família sobre resíduos dos serviços de saúde. Revista Brasileira de Enfermagem. v.65, n.4, p.645652, 2012.

SANTOS, N. M. Gerenciamento integrado de resíduos sólidos: estudo de caso no Instituto Butantan. Dissertação (Mestrado Profissional em Ambiente, Saúde e Sustentabilidade). Faculdade de Saúde Pública, Universidade de São Paulo, São Paulo, SP, Brasil. 2015.

Teixeira, M. V; ECHEVARRÍA-GUANILO, M. E; KNUTH, F. G; CEOLIN, T. Avaliação da Gestão dos Resíduos em Unidades Básicas de Saúde de um Município Sul- Brasileiro. Revista Fundamental Care Online. v.10, n.3, p. 824-831, 2018.

WHO. World Health Organization. Health Systems Financing the path to universal coverage, Genebra, 2010. Disponível em: http://www.who.int/ whr/2010/10_summary_en.pdf. Acesso em: $18 / 05 / 19$ 